\title{
Oral mini-pulse steroid therapy in severe chronic urticaria
}

\author{
Mohammad Abid Keen', Faizan Younus Shah² \\ ${ }^{1}$ Consulting dermatologist, Dr Abid's Skincare and laser center, Anantnag, India, ${ }^{2}$ Senior Resident, Department of \\ Dermatology, STDs \& Leprosy, Government Medical College, Srinagar, India
}

Corresponding author: Mohammad Abid Keen, MD, E-mail: keenabid31@ gmail.com

\begin{abstract}
Background: Treatment of chronic urticaria can be difficult at times. The present study aimed to evaluate the efficacy of oral mini-pulse (OMP) therapy with methylprednisolone in management of severe chronic urticaria (CU). Methods and material: 100 patients with severe chronic urticaria, not controlled with maximum dose of a second generation antihistamine, were enrolled in the study after an informed written consent. All patients were treated with methylprednisolone $16 \mathrm{mg}$ tablet on two consecutive days of a week for 2 months along with levocetirizine $5 \mathrm{mg}$ tablet once daily. All patients were reviewed at $0,2,4$, and 8 weeks with urticaria activity score (UAS). Results: The study comprised of 100 patients (33 males and 67 females) with severe chronic urticaria. 29 patients (29\%) had raised TSH levels while Autologous Serum Skin Test was positive in 37 patients. Mean UAS in patients treated with OMP was 5.76 at baseline which reduced to 0.6 at the end of treatment period. Conclusion: Mean UAS showed a significant decline following OMP therapy with methylprednisolone. Most of the patients maintained the benefits of therapy at the end of follow up period of 4 months.
\end{abstract}

Key words: Chronic urticaria; Oral mini-pulse (OMP) therapy; Methylprednisolone; Urticaria activity score

\section{INTRODUCTION}

Urticaria is a fairly common condition characterized by transient swellings of the skin. This could be an extremely disabling and difficult-to-treat condition. Chronic urticaria $(\mathrm{CU})$ is defined as urticaria persisting daily or almost daily for more than six weeks. It causes severe impairment on the quality of life [1]. CU includes physical urticaria, chronic "idiopathic" urticaria (CIU), chronic autoimmune urticarial (CAU) and urticarial vasculitis. It is important to recognize patients with physical urticaria, as the need for investigation and treatment modalities differ from patients with CIU or urticarial vasculitis. If we eliminate physical urticaria and urticarial vasculitis from all patients with $\mathrm{CU}$, the remainder can be divided into CAU (45\%) and CIU (55\%). The autoimmune subgroup is associated with the IgG anti-IgE receptor alpha subunit in $35-40 \%$ of the patients, and IgG anti-IgE in an additional 5-10\%. These autoantibodies have been shown to activate blood basophils and cutaneous mast cells in vitro, with augmentation of basophil activation by complement and release of $\mathrm{C} 5 \mathrm{a}$.

Depending upon the severity of the disease and response to various medicines, the drug therapy can be considered at various levels. The treatment can be started with a second generation non-sedating (or less sedating) antihistamines like cetirizine, loratadine, fexofenadine, astemizole, mizolastine, ebastine etc. In case of inadequate response, the dose can be increased upto four fold. Systemic corticosteroids constitute second line therapy for chronic urticaria. The use of systemic corticosteroids in the treatment of urticaria is a controversial issue. Short courses of systemic steroids (20-30 mg of prednisolone) can be given in resistant cases of chronic urticaria that have not responded to $\mathrm{Hl}$ antihistamine [2]. The efficacy of corticosteroid therapy is high, but long term therapy cannot be promoted because of 
known adverse effects, such as diabetes mellitus, hypertension, osteoporosis and gastrointestinal bleeding. Prolonged treatment of chronic urticaria with oral corticosteroids should usually be avoided except in disabling delayed or pressure urticaria and urticarial vasculitis, which are usually nonresponsive to antihistamines. Immunotherapy is considered as a third line therapy and could be tried in patients with severe refractory autoimmune urticaria. Various immunosuppressants that have been tried in severe chronic urticaria include cyclosporine, methotrexate, sulphasalazine, dapsone, cyclophosphamide, and intravenous immunoglobulin [3-8]. In a recent placebo controlled study autohaemotherapy has shown a beneficial role in ASST positive chronic urticaria patients [9].

In order to evaluate the efficacy of oral methylprednisolone mini-pulse (OMP) in severe chronic urticaria, we conducted a study on 100 patients with CIU and CAU, who were treated with oral methylprednisolone mini-pulse (OMP).

\section{METHODS}

This study was carried out at our private dermatology centre over a period of one year, from October 2018 to September 2019. 100 consecutive diagnosed cases of severe chronic urticaria, aged between 15 years and 65 years were enrolled in the study after obtaining an informed consent. All the 100 patients had severe chronic idiopathic urticaria of duration ranging from 2 months to 120 months (mean duration $=19.14$ \pm 26.03 months) which was not controlled with maximum dose of a second generation antihistamine. Urticarial vasculitis was ruled out by excluding cases wherein the lesions were not evanescent and remained persistent for a duration exceeding 24 hours.

Exclusion criteria were physical urticaria, urticarial vasculitis, pregnant or lactating women, history of gastritis and a history of sensitivity to aspirin or NSAIDs. Routine investigations such as complete blood count, blood sugar, thyroid-stimulating hormone (TSH), and urine examination were performed in all the patients.

Autologous Serum Skin Test (ASST) was performed in all the patients before putting them on treatment. ASST was performed as follows: About $5 \mathrm{ml}$ venous blood was collected in a sterile vacutainer and allowed to clot at room temperature for 30 minutes. Serum was sent to the clinical laboratory for centrifugation at $2000 \mathrm{rpm}$ for 15 minutes and $0.05 \mathrm{ml}$ of autologous serum (test) was then injected intradermally using a $1 \mathrm{ml}$ insulin syringe (30 gauge needle) on the right forearm $2 \mathrm{~cm}$ below the cubital fossa and similarly 0.05 $\mathrm{ml}$ of $0.9 \%$ sterile normal saline (control) was injected intradermally to the left forearm. A serum induced erythematous wheal with a diameter of $1.5 \mathrm{~mm}$ more than the saline induced response within 30 minutes was taken as a positive test. In all subjects, antihistamine and steroids were withdrawn at least 72 hours and one week prior to skin test, respectively.

Investigations such as IgE, cold agglutinin, Anti-TPO antibodies, Anti TG antobodies were not done due to high cost in a private clinic setup.

In all these patients, we started treatment with methylprednisolone $16 \mathrm{mg}$ tablet, given twice a week after breakfast for 2 months along with levocetirizine 5 mg tablet daily at bed time. The patients were reviewed at $0,2,4,6$ and 8 weeks with urticaria activity score. The urticaria activity score consisted of the sum of the wheal number score and the itch severity score [10]. The blood pressure and weight of these patients was recorded initially and then monitored on monthly basis. Patients were asked for and examined for side effects such as weight gain, hypertension, gastritis, acneiform eruption, striae, and Cushingoid features. The patients were followed up monthly for 4 months after stopping the steroids and relapses were noted.

\section{RESULTS}

The present study comprised of 100 patients $(33$ males and 67 females) with severe chronic urticaria. The age of patients ranged from 15 to 65 years, with a mean age of $33.99 \pm 11.79$ years. Age and sex distribution of patients is given in Table 1 .

Duration of urticaria in these patients ranged from 2 months to 120 months, with a mean duration of $19.14 \pm 26.03$ months, which was not controlled with maximum dose of a non-sedating second generation antihistamine.

Investigations revealed that 29 patients $(29 \%)$ had raised TSH levels. Physician's opinion was taken in these patients regarding thyroxine replacement. Mean TSH levels in these patients was $5.01 \pm 4.36$. Autologous Serum Skin Test (ASST) was positive in 37 patients (6 males and 31 females). ASST positivity 
in CU was more commonly encountered in females $(46.97 \%, \mathrm{n}=31 / 66)$ as compared to males $(18.18 \%, \mathrm{n}$ $=6 / 33$ ) and the difference was found to be statistically significant using student's t-test ( $\mathrm{p}$-value $=0.006$ ) . Thus, women were more likely to have an autoimmune etiology in CU as compared to males.

Mean urticaria activity score in patients treated with mini-pulse was 5.76 at baseline ( 0 weeks). At the end of 2 weeks, mean scores was 2.51 . At the end of 4 weeks, mean urticaria activity score came down to 1.79. Mean urticaria activity score at the end of 6 weeks and 8 weeks was found to be decreased to 1.14 and 0.6 respectively.

Side effects as the result of treatment were seen in the form of epigastric pain (13 patients), acne (2 patients), weight gain (l patient) and hypertention (1 patient).

Among our study group of 100 patients, 12 (12\%) relapsed, with worsening of symptoms after stopping steroids. Rest 88 (88\%) patients (30 males and 58 females) remained well controlled and the remission was maintained till follow up period of four months after stopping steroids.

\section{DISCUSSION}

Urticaria is a fairly common condition characterized by transient swellings of the skin. This could be an extremely disabling and difficult-to-treat condition. Conventionally chronic urticaria is defined as the repeated occurrence of daily or almost daily cutaneous wheals accompanied by redness and itching for more than 6 weeks. Chronic urticaria has multifactorial aetiologies including intolerance to food or drugs, infectious diseases, and autoimmune processes. Chronic urticaria has a wide spectrum of clinical presentations and despite our best efforts no cause may be found in the majority of cases. Depending upon the various aggravating factors and the presence of circulating auto antibodies, chronic urticaria can be further divided in three subgroups:

\begin{tabular}{llcc}
\multicolumn{4}{l}{ Table 1: Demographic characteristics of patients } \\
\hline S.No & Demographic characteristic & Group & Percentage \\
\hline 1 & Age group & $11-20$ years & $12 \%$ \\
& & $21-30$ years & $37 \%$ \\
& & $31-40$ years & $21 \%$ \\
& & $41-50$ years & $21 \%$ \\
& & $51-60$ years & $8 \%$ \\
& \multirow{2}{*}{ Gender } & $61-70$ years & $1 \%$ \\
& & Male & $33 \%$ \\
& & Female & $67 \%$ \\
\hline
\end{tabular}

(c) Our Dermatol Online e.2021
Chronic urticaria with potential provoking factors (including drugs, food, food additives, infections, parasitic infestations, collagen and endocrine disorders, dermatological disorders etc).

Autoimmune urticaria (about 30 to $50 \%$ patients with chronic urticaria have circulating functional auto antibodies against the high affinity $\operatorname{IgE}$ receptor FCeRIa or against IgE).

Chronic idiopathic urticaria (even after evaluation for evidence of autoimmunity and other provoking factors, approximately $50 \%$ cases of chronic urticaria remain unexplained and are categorized as chronic idiopathic urticaria).

An affected patient often goes from one dermatologist to another in pursuit of a cure. Treatment for all forms of CU should include a sound, basic lifestyle. These include avoidance of stress, overtiredness, alcohol, NSAIDS, and tight fitting garments. Nocturnal pruritus can be reduced by tepid showering and keeping the ambient temperature of the bedroom cool. Cooling lotions such as calamine with $1 \%$ menthol are popular with patients.

Antihistamines are the first line treatment for all patients with chronic urticaria. Treatment is generally started with nonsedating second generation antihistamine with up-dosing to four-fold, in case of inadequate response. Nonsedating second generation $\mathrm{Hl}$ antihistamines include loratadine, cetirizine, terfenadine, and mizolastine, desloratadine, levocetirizine, and fexofenadine. H2 antihistamines include cimetidine, ranitidine, famotidine and nizatadine. It has been seen that combination of antihistamines often helps the patient. One study from secondary referral center found that $40 \%$ patients did not respond to antihistamines [11].

Doxepin is the second line of treatment if antihistamines do not work and has shown superiority over diphenhydramine in various studies [12]. Montelukast is a leukotriene receptor antagonist and works well for aspirin-sensitive urticarial [13]. A study found montelukast monotherapy ineffective in CIU when compared with cetirizine [14].

Corticosteroids can be considered in chronic urticaria where antihistamines in high doses failed to show desired result. They should be used cautiously and for only short periods due to their side effect profile. Pasricha and Khaitan et al. have devised a new 
therapeutic regime called OMP therapy for reducing the side effects of corticosteroids. The OMP regime had been primarily designed for treating patients having fast spreading/extensive vitiligo to achieve the therapeutic results with the minimum of side effects [15]. Similar therapy is used for treatment of lichen planus and alopecia totalis $[16,17]$. Systemic corticosteroids act at multiple levels, they suppress the formation of antibodies, they have a strong anti-inflammatory effect, and in addition, are known to decrease the production of histamine-releasing factors [18]. Steroids should be used very cautiously in patients with diabetes, hypertension, and osteoporosis.

In this study, we found that $29 \%$ of our patients suffering from CU had a raised TSH level $(>5.5 \mu \mathrm{U} / \mathrm{ml})$. This was much higher than the prevalence of hypothyroidism reported as $10.95 \%$ in Indian population based on similar criteria [19]. This is expected as a relation of hypothyroidism and autoimmune thyroid disorder with chronic urticaria has been reported in literature $[20,21]$.

Association with an underlying autoimmune etiology was also more likely to be seen in case of females as compared to males and this had a statistical relevance. This was expected as autoimmune disorders are known to be more common among females [22]. It could not be established unequivocally whether the autoimmune etiology encountered in our cases was related to autoimmune thyroiditis as anti-TPO antibody testing could not be performed.

Mean urticaria activity score showed a significant decline following institution of OMP therapy with methylprednisolone with a decline of more than $50 \%$ within 2 weeks of institution of treatment. The mean UAS went on to decrease by $89.58 \%$ at the end of 8 week treatment period. Most of the patients maintained the benefits of therapy at the end of follow up period of 4 months with relapse being seen in a minimal proportion (12\%) of patients.

Treatment of chronic urticaria can pose a major challenge to the physician. Many patients with chronic urticaria have at least partial response to antihistamines, but there are a proportion of patients that do not respond; thus requiring a more aggressive treatment with different types of immunomodulatory agents. Thus, dermatologists must be knowledgeable about the implementation of correct treatment. There is a wide range of non-pharmacological and pharmacological options and there is no universal solution, therefore the most appropriate treatment should be tailored individually for each patient.

In summary, on the basis of the outcome of our study, oral methylprednisolone mini-pulse therapy is a viable options in treating patients with severe chronic urticaria not responding to first and second lines of treatments.

\section{CONCLUSION}

Chronic urticaria is one of the most vexing management problems that dermatologists face and still remains a therapeutic dilemma. Most of the therapeutic modalities used in the treatment of severe chronic urticaria are immunosuppressants such as methotrexate, cyclosporine, or omalizumab. However their use is limited by factors like need for prolonged therapy, related side effects and toxicity, presence of co-morbid disorders, need for elaborate laboratory testing, regular patient monitoring, and high cost of therapy. Because of these reasons, immunosupressive drugs can't be used routinely in an Indian setting. So, corticosteroids in oral mini-pulse therapy can further broaden our pharmacologic approach and be a ray of hope in the management of patients with severe refractory chronic urticaria.

\section{Statement of Human and Animal Rights}

All the procedures followed were in accordance with the ethical standards of the responsible committee on human experimentation (institutional and national) and with the 2008 revision of the Declaration of Helsinki of 1975.

\section{Statement of Informed Consent}

Informed consent for participation in this study was obtained from all patients.

\section{REFERENCES}

1. Pherwani AV, Bansode G, Gadhia S. The impact of chronic urticaria on the quality of life in Indian patients. Indian J Dermatol. 2012;57:110-3.

2. Greenberger, P.A. Chronic urticaria: new management options. World Allergy Organ J. 2014;7:1-6.

3. Kaplan AP. Treatment of chronic spontaneous urticaria. Allergy Asthma Immunol Res. 2012;4:326-31.

4. Sharma VK, Singh S, Ramam M, Kumawat M, Kumar R. A randomized placebo-controlled double-blind pilot study of methotrexate in the treatment of H1 antihistamine-resistant chronic spontaneous urticaria. Indian J Dermatol Venereol Leprol. 2014;80:122-8.

5. Asero R, Tedeschi A, Cugno M. Treatment of refractory chronic urticaria: current and future therapeutic options. Am J Clin Dermatol. 2013;14:481-8. 
6. Liang SE, Hoffmann R, Peterson E, Soter NA. Use of dapsone in the treatment of chronic idiopathic and autoimmune urticaria. JAMA Dermatol. 2019;155:90-5.

7. Kim J, Chan JJ. Cyclophosphamide in dermatology. Australas J Dermatol. 2017;58:5-17.

8. Watkins C, Peiris E, Saleh H, Krishnaswamy G. Intravenous immunoglobulin as a potential therapy for refractory urticaria - a review. Inflamm Allergy Drug Targets. 2012;11:375-81.

9. Zhang L, Xiao X, Hui R, Shi Y, Deng Y, Zheng H, et al. Autologous whole-blood or autologous serum acupoint injection therapy for chronic urticaria: A systematic review protocol. Medicine (Baltimore). 2019;98:e16127.

10. de Silva NL, Damayanthi H, Rajapakse AC, Rodrigo C, Rajapakse S. Leukotriene receptor antagonists for chronic urticaria: a systematic review. Allergy Asthma Clin Immunol. 2014;10:24.

11. Tang N, Mao MY, Zhai R, Chen X, Zhang JL, Zhu W, et al. Clinical characteristics of urticaria in children versus adults. Zhongguo Dang Dai Er Ke Za Zhi. 2017;19:790-5.

12. Adhya Z, Karim Y. Doxepin may be a useful pharmacotherapeutic agent in chronic urticaria. Clin Exp Allergy. 2015;45:1370.

13. Wan KS, Chang YS. Efficacy of leukotriene receptor antagonist with anti-H1 receptor antagonist plus anti-H2 receptor antagonist for treatment of refractory chronic idiopathic urticaria. J Dermatolog Treat. 2014;25:459-61.

14. Godse KV. Oral montelukast monotherapy is ineffective in chronic idiopathic urticaria: A comparison with oral cetirizine. Indian J Dermatol Venereol Leprol. 2006;72:312-4.

15. Kanwar AJ, Mahajan R, Parsad D. Low-dose oral mini-pulse dexamethasone therapy in progressive unstable vitiligo. J Cutan Med Surg. 2013;17:259-68.
16. Jang YH, Kim SL, Lee KC, Kim MJ, Park KH, Lee WJ, et al A comparative study of oral cyclosporine and betamethasone minipulse therapy in the treatment of alopecia areata. Ann Dermatol. 2016;28:569-74.

17. Ramesh M, Balachandran C, Shenoi SD, Rai VM. Efficacy of steroid oral mini-pulse therapy in lichen planus: An open trial in 35 patients. Indian J Dermatol Venereol Leprol 2006;72:156-7.

18. Asero R, Pinter E, Marra AM, Tedeschi A, Cugno M, Marzano AV. Current challenges and controversies in the management of chronic spontaneous urticaria. Expert Rev Clin Immunol. 2015;11:1073-82.

19. Unnikrishnan AG, Kalra S, Sahay RK, Bantwal G, John M, Tewari N Prevalence of hypothyroidism in adults: An epidemiological study in eight cities of India. Indian J Endocrinol Metab. 2013;17:647-52.

20. Kasumagic-Halilovic E, Beslic N, Ovcina-Kurtovic N. Thyroid autoimmunity in patients with chronic urticaria. Med Arch. 2017;71:29-31.

21. Najafipour M, Zareizadeh M, Najafipour F. Relationship between Chronic urticaria and autoimmune thyroid disease. J Adv Pharm Technol Res. 2018;9:158-61.

22. Fairweather D, Frisancho-Kiss S, Rose NR. Sex differences in autoimmune disease from a pathological perspective. Am J Pathol. 2008;173:600-9.

Copyright by Mohammad Abid Keen et al. This is an open access article distributed under the terms of the Creative Commons Attribution License which permits unrestricted use, distribution, and reproduction in any medium, provided the original author and source are credited.

Source of Support: Nil, Conflict of Interest: None declared. 